\title{
Protection of smart substation based on WLAN complies with IEC 61850 using traveling wave analysis
}

\begin{abstract}
Fast protection of distribution substation based on traveling wave is becoming a reality today. The transient traveling wave is mostly used in protection to determine the faulted line and the fault location. The advantages of signal of traveling wave includes immunity to system oscillation, transition resistance, CT saturation, and neutral point operation modes. In this article, a detailed study for analyzing the performance of using WLAN for real time protection based on the transient traveling wave. The high sampling frequency needed for traveling wave causes blockage in communication in the process bus. Now a packing of multi samples with suitable compression techniques in Merging Unit (MU), or using feature extraction with Hilbert-Huang transform with WLAN compliance with IEC 61850 has been tested. Further, this article presents the modeling and simulation of a WLAN communication network for an automation system using the precepts of IEC 61850, which is currently becoming a trend in Substation Automation System (SAS) specification. This article also investigates the impact of impulsive and interface noise on WLAN performance. Finally, the article advocates the wide application of traveling waves in the digital substations based on the IEC 61850 protocol.
\end{abstract}

Keyword: IEC 61850; Traveling wave; Packing; Feature extraction; WLAN 